\title{
Factors Affecting the Discharge Destination of Patients With Spinal Bone Metastases
}

\author{
Yoshiteru Akezaki, RPT, $\mathrm{PhD}^{1}$, Eiji Nalkata, $\mathrm{MD}, \mathrm{PhD}^{2}$, \\ Masato Kikuuchi, OTR ${ }^{3}$, Shinsuke Sugihara, $\mathrm{MD}, \mathrm{PhD}^{3}$ \\ ${ }^{1}$ Division of Physical Therapy, Kochi Professional University of Rehabilitation, Kochi; \\ ${ }^{2}$ Department of Orthopaedic Surgery, Okayama University, Okayama; \\ ${ }^{3}$ Department of Rehabilitation Medicine, National Hospital Organization Shikoku Cancer Center, Ehime, Japan
}

\begin{abstract}
Objective To investigate the factors affecting the discharge destination of patients with spinal bone metastases. Methods We reviewed the medical records of patients admitted to our institute with a diagnosis of skeletalrelated events secondary to malignant disease. Exclusion criteria comprised decreased cognitive function and hypercalcemia, brain metastasis, peritoneal dissemination, and pleural dissemination. The factors examined included the discharge destination, age, sex, the Barthel Index (BI) at admission and discharge, pain at admission and discharge, number of co-resident household members, length of hospital stay, treatment strategy, spinal instability neoplastic score, vertebral body collapse, spinal level of bone metastases, and motor paralysis. For the discharge destination, patients at discharge were grouped into two categories. The home group included patients discharged to their own homes, and the non-home group included patients discharged to other hospitals.

Results Of 140 patients, the home group comprised 120 patients and the non-home group comprised 20 patients. Activities of daily living (ADL) and pain at rest and during motion improved significantly in the home group, whereas only pain at rest and during motion improved significantly in the non-home group. The results indicated that discharge BI and motor paralysis were the best predictors of the discharge destination; a BI cut-off value of 72.5 predicted discharge to home.

Conclusion This study showed that the ADL level on discharge and motor paralysis affected the discharge destination of patients with spinal bone metastases. These results are likely to be helpful in predicting the discharge destination of patients with spinal bone metastases.
\end{abstract}

Keywords Cancer, Pain, Activities of daily living, Spine, Motor paralysis

Received May 31, 2019; Accepted September 3, 2019

Corresponding author: Yoshiteru Akezaki

Division of Physical Therapy, Kochi Professional University of Rehabilitation, Otu-1139-3, Takaoka-Machi, Tosa, Kochi 781-1102, Japan. Tel: +81-88850-2311, Fax: +81-88-850-2323, E-mail: akezakiteru@yahoo.co.jp

ORCID: Yoshiteru Akezaki (https://orcid.org/0000-0002-9613-9781); Eiji Nakata (https://orcid.org/0000-0002-5971-4142); Masato Kikuuchi (https:// orcid.org/0000-0003-4994-497X); Shinsuke Sugihara (https://orcid.org/0000-0002-9460-3982).

(c) This is an open-access article distributed under the terms of the Creative Commons Attribution Non-Commercial License (http://creativecommons.org/ licenses/by-nc/4.0) which permits unrestricted noncommercial use, distribution, and reproduction in any medium, provided the original work is properly cited. Copyright $\odot 2020$ by Korean Academy of Rehabilitation Medicine 


\section{INTRODUCTION}

The most common site of bone metastases is the spine, and the presence of spinal metastases is frequently associated with malignant primary disease at an advanced stage $[1,2]$. A diagnosis of spinal metastases is made during a patient's life in $5 \%-10 \%[3,4]$ of cases involving systemic cancer, but studies have found that up to $90 \%$ of patients who die of cancer had spinal metastases on postmortem examination [5]. In terms of the sequelae of spinal metastases, spinal cord compression is the most serious, affecting $20 \%$ of patients [6]. The most common region of the spine affected due to metastases is the thoracic spine, followed by the lumbar and cervical regions $[5,7,8]$. Bone metastases cause significant pain and morbidity, and they are associated with a variety of skeletalrelated events (SREs), such as pathological fractures, a need for radiation or bone surgery, spinal cord compression, and hypercalcemia of malignancy. Paralysis and prolonged bed rest after SREs result in decreased physical function, which can then decrease patients' activities of daily living (ADL) and quality of life (QOL).

Research has demonstrated the benefits of rehabilitation for patients with cancer. In a study by Bunting et al. [9], patients transferred to a rehabilitation hospital following surgical stabilization of their pathological fractures were found to have significantly improved mobility and ADL. In a study of patients with metastatic spinal cord compression, Tang et al. [10] showed that functional outcomes were significantly improved through participation in a rehabilitation program, based on assessment using the Functional Independence Measure. Returning patients to their homes is the goal of most rehabilitation programs so that patients can live out their lives in a manner that is as close to normal as possible.

Many studies have examined patients with stroke in relation to the discharge destinations of inpatients. Denti et al. [11] found that the strongest predictor of the discharge destination for elderly patients with stroke was the admission ADL level. Okuno et al. [12] also found that the ADL level on discharge was also an effective predictor of the discharge status in patients with stroke. Additionally, improved Kenny scores have been found to be associated with discharge home for patients with cancer-related pathological limb fractures [9]. However, with respect to patients with SREs, few studies have investigated the ADL level needed for patients to return home.

The purpose of this study was to investigate factors related to the discharge destination of patients with spinal bone metastases

\section{MATERIALS AND METHODS}

\section{Study design}

This was a retrospective, observational study of the discharge destination in patients with spinal bone metastases.

\section{Participants}

The medical records of patients admitted to our institute with a diagnosis of SRE secondary to malignant disease between September 2011 and March 2013 were reviewed. Patients included in our study had SREs such as pathological fractures, a need for radiation therapy to the bone, or spinal cord compression. Exclusion criteria comprised decreased cognitive function and hypercalcemia, brain metastasis, peritoneal dissemination, and pleural dissemination.

\section{Clinical parameters}

We investigated the following factors: the discharge destination, age, sex, the Barthel Index (BI) at admission and discharge, pain at admission and discharge, the number of co-resident household members, length of hospital stay, treatment strategy, the spinal instability neoplastic score (SINS) [13], vertebral body collapse, spinal level of bone metastases, and motor paralysis.

\section{Ethical approval statement}

All procedures undertaken as part of this study involving human participants were performed under an approved protocol and in accordance with the ethical standards of Shikoku Cancer Center Ethics Committee (Approval No. 114) and with the 1964 Helsinki Declaration and its later amendments or comparable ethical standards.

\section{Discharge destination}

Concerning the discharge destination, patients at discharge were grouped into two categories. Patients who were discharged to their homes constituted the home group, and those who were discharged to other hospitals 
constituted the non-home group.

\section{Activities of daily living, pain, and treatment strategy}

The BI [14] is an ordinal scale used to measure ADL performance (i.e., feeding oneself, bathing, dressing, grooming, and the ability to move) on a scale of $0-100$ (0, very dependent; 100, independent). All patients were assessed within 48 hours of admission and within 48 hours before discharge.

Pain was assessed using a Numerical Rating Scale (NRS), with 0 representing 'no pain' and 10 representing 'pain as bad as you can imagine'. Pain was evaluated at rest and during motion. Pain evaluation in this study was limited to spinal metastasis-related pain.

Patients treated with anticancer drugs were categorized as belonging to an anticancer drug group, and patients who received palliative treatment were categorized as belonging to a palliative treatment group.

\section{Determination of length of hospital stay and discharge destination}

With respect to determining the length of hospital stay and the discharge destination, a doctor consulted with a patient and the patient's family, taking into consideration the patient's general condition, their ADL, family situation, and the need for social resources such as homevisiting nursing services, home-visit rehabilitation, and home helpers. During hospitalization, doctors, nurses, physiotherapists, occupational therapists, and pharmacists conducted multiple conferences and shared information. The length of hospital stay and discharge destination were ultimately determined through giving priority to the wishes of patients and their families.

\section{Vertebral body collapse}

The presence or absence of vertebral body collapse was determined using the SINS for vertebral collapse evaluation. Patients with $0-1$ points were classified as having no vertebral body collapse, and those with 2-3 points were classified as having vertebral body collapse.

\section{Spinal level of bone metastases}

The spinal level of bone metastases in the patients was classified according to three sites, namely, cervical vertebrae, thoracic vertebrae, and lumbar vertebrae.

\section{Motor paralysis}

Motor paralysis was assessed using the Frankel scale. Patients with grades A, B, C, and D were classified as having motor paralysis, while those with grade $\mathrm{E}$ were classified as not having motor paralysis.

\section{Rehabilitation program}

The rehabilitation program comprised muscle strengthening exercises, involving squatting and calf-raising exercises; stretching, involving the upper limb, lower limb, and trunk; and ADL, including standing up and walking. Rehabilitation was undertaken according to the degree of independence in performing ADL and in relation to the pain level. Exercise intensity was performed at 11-13 on the Borg Scale [15]. The exercise frequency was 5 days a week for 20-40 minutes.

\section{Statistical analysis}

Changes in ADL and pain at admission and discharge were analyzed using the paired t-test in relation to the home and non-home groups.

Univariate analysis was carried out using Student t-test, the chi-square test, and the Mann-Whitney U test to identify factors associated with the discharge destination of patients with spinal bone metastases. For items found to have p-values $<0.2$ on univariate analysis, logistic regression analysis was used to identify the best independent predictor of the discharge destination of patients with spinal bone metastases. The usefulness of the variables for making predictions in the home group was investigated using a receiver operating characteristic (ROC) curve, and the cut-off value necessary for the home group was determined and evaluated in terms of the sensitivity, the false-positive rate (1-specificity), the predictive accuracy, and the positive predictive value.

Overall survival (OS) was calculated from the date of admission to our institute with a diagnosis of SRE until death from any cause. The observations of this study were censored on the date the patient was last known to be alive, by a doctor or nurse. The follow-up cut-off point was determined as November 30, 2017. Survival probabilities were estimated using Kaplan-Meier analysis, and significant differences were analyzed using a log-rank test. Statistical analysis was performed using SPSS Statistics version 22.0 (IBM, Tokyo, Japan). The results were defined as being significant when the possibility of error 
(p) was $<5 \%$.

\section{RESULTS}

\section{Clinical and sociodemographic characteristics}

The participants comprised 140 patients, with an average \pm standard deviation age at the time of the study of $66.4 \pm 9.7$ years. The type of primary cancer involved is shown in Table 1. The tumor treatment options included radiation therapy in 133 patients, and no patients underwent surgery.

The home group included 120 of the 140 patients, and the non-home group included 20 patients. The length of hospital stay was $26.2 \pm 18.7$ days in the home group and $36.0 \pm 26.0$ days in the non-home group.

Table 1. Type of primary cancer of patients

\begin{tabular}{|l|c|}
\hline \multicolumn{1}{|c}{ Characteristic } & Number of cases \\
\hline Lung cancer & 40 \\
\hline Breast cancer & 31 \\
\hline Prostate cancer & 20 \\
\hline Rectal cancer & 15 \\
\hline Gastric cancer & 9 \\
\hline Hepatocellular cancer & 6 \\
\hline Renal cell cancer & 4 \\
\hline Esophageal cancer & 3 \\
\hline Bladder cancer & 2 \\
\hline Pancreatic cancer & 2 \\
\hline Paget disease of the breast & 1 \\
\hline Gastric carcinoid & 1 \\
\hline Uterine cancer & 1 \\
\hline Gallbladder cancer & 1 \\
\hline Bile duct cancer & 1 \\
\hline Mesothelioma & 1 \\
\hline Rectal carcinoid & 1 \\
\hline Endothelioma & 1 \\
\hline
\end{tabular}

Changes in ADL and pain at admission and discharge

Changes in ADL and pain on admission and at discharge are shown in Table 2. Pain was considered as metastasis-related pain. ADL and pain at rest and during motion improved significantly in the home group, whereas only pain at rest and during motion improved significantly in the non-home group.

\section{Factors affecting the discharge destination}

The results of the univariate analysis are shown in Table 3. The BI at admission and discharge, pain at rest on admission, pain during movement at admission and discharge, the SINS, and the degree of motor paralysis differed between the two groups $(\mathrm{p}<0.2)$. Using logistic regression analysis, the discharge $\mathrm{BI}$ and motor paralysis were found to be significant predictors of discharge to home (both, $\mathrm{p}<0.05)$ (Table 4).

\section{Target value required for home return}

Fig. 1 shows the ROC curves of the BI at discharge for the home group. The area under the curve was 0.843 , with a standard error of 0.059 and a $95 \%$ confidence interval of $0.727-0.959$. With a BI cut-off value of 72.5 , the sensitivity was $88.3 \%$, the false-positive rate (1-specificity) was $25.0 \%$, the predictive accuracy was $86.4 \%$, and the positive predictive value was $95.5 \%$.

\section{Kaplan-Meier survival curves of the home and non- home groups}

The Kaplan-Meier survival curves are shown in Fig. 2. In the home group, the median OS was 11.5 months, with 3 - and 6 -month survival rates of $82.5 \%$ and $67.5 \%$, respectively. In the non-home group, the median OS was 9.1 months, with 3- and 6-month survival rates of $70.0 \%$ and $60.0 \%$, respectively $(\mathrm{p}=0.573)$. No significant differences between the groups were observed (Fig. 2).

Table 2. Results of activities of daily living and pain at admission and discharge

\begin{tabular}{lccccccc}
\hline & \multicolumn{3}{c}{ Home group $(\mathbf{n = 1 2 0})$} & & \multicolumn{3}{c}{ Non-home group $(\mathbf{n = 2 0})$} \\
\cline { 2 - 3 } \cline { 6 - 7 } & Admission & Discharge & p-value & & Admission & Discharge & p-value $^{\text {a) }}$ \\
\hline Barthel Index & $78.9 \pm 26.5$ & $91.4 \pm 13.7$ & $<0.0001$ & & $48.0 \pm 34.7$ & $53.0 \pm 32.1$ & 0.278 \\
Rest pain & $1.1 \pm 2.2$ & $0.1 \pm 0.6$ & $<0.0001$ & & $1.7 \pm 3.0$ & $0.1 \pm 0.2$ & 0.007 \\
Motion pain & $2.7 \pm 3.3$ & $0.6 \pm 1.2$ & $<0.0001$ & & $4.3 \pm 3.6$ & $0.3 \pm 0.6$ & $<0.0001$ \\
\hline
\end{tabular}

Values are presented as mean \pm standard.

${ }^{\text {a)}}$ Paired t-test. 
Table 3. Comparison of variables between the home group and non-home group

\begin{tabular}{|c|c|c|c|}
\hline Variable & Home group $(n=120)$ & Non-home group $(\mathbf{n}=\mathbf{2 0})$ & p-value \\
\hline Age (yr) & $66.2 \pm 8.7$ & $67.8 \pm 14.2$ & 0.812 \\
\hline Sex & & & 0.631 \\
\hline Male & 61 & 11 & \\
\hline Female & 59 & 9 & \\
\hline \multicolumn{4}{|l|}{ Barthel Index (score) } \\
\hline At admission & $78.9 \pm 26.5$ & $48.0 \pm 34.7$ & $<0.0001$ \\
\hline At discharge & $91.4 \pm 13.7$ & $53.0 \pm 32.1$ & $<0.0001$ \\
\hline \multicolumn{4}{|l|}{ Pain in rest (score) } \\
\hline At admission & $1.1 \pm 2.2$ & $1.7 \pm 3.0$ & 0.128 \\
\hline At discharge & $0.1 \pm 0.6$ & $0.1 \pm 0.2$ & 0.847 \\
\hline \multicolumn{4}{|l|}{ Pain during movement (score) } \\
\hline At admission & $2.7 \pm 3.3$ & $4.3 \pm 3.6$ & 0.033 \\
\hline At discharge & $0.6 \pm 1.2$ & $0.3 \pm 0.6$ & 0.179 \\
\hline Number of family members & $1.6 \pm 1.2$ & $1.3 \pm 1.0$ & 0.348 \\
\hline Treatment strategy & & & 0.001 \\
\hline Anticancer drug & 88 & 7 & \\
\hline Palliation treatment & 32 & 13 & \\
\hline Spinal instability neoplastic score & $6.9 \pm 2.6$ & $8.5 \pm 2.3$ & 0.011 \\
\hline Vertebral body collapse & & & 0.464 \\
\hline Yes & 72 & 14 & \\
\hline No & 48 & 6 & \\
\hline Spine level of bone metastases & & & 0.572 \\
\hline Cervical & 13 & 2 & \\
\hline Thoracic & 56 & 8 & \\
\hline Lumbar & 51 & 10 & \\
\hline Motor paralysis & & & $<0.0001$ \\
\hline Yes & 4 & 7 & \\
\hline No & 116 & 13 & \\
\hline \multicolumn{4}{|l|}{ Frankel scale } \\
\hline A & 0 & 2 & \\
\hline $\mathrm{B}$ & 0 & 0 & \\
\hline $\mathrm{C}$ & 0 & 1 & \\
\hline $\mathrm{D}$ & 4 & 4 & \\
\hline $\mathrm{E}$ & 116 & 13 & \\
\hline
\end{tabular}

Values are presented as mean \pm standard or number.

\section{DISCUSSION}

Numerous reports have shown that ADL on admission and at discharge were significant predictors of the discharge destination in patients with stroke $[11,12,16,17]$. In patients with cancer-related pathological limb fractures, those discharged to their homes were reported to have improved Kenny scores [9]. In the present study, the home group showed improved ADL and pain at rest and during motion, whereas the non-home group only improved significantly in terms of pain at rest and during motion. The logistic regression analysis showed that the discharge BI and motor paralysis were critical factors for the home group. These results demonstrated that 
Table 4. Logistic regression analysis for prediction of discharge destination

\begin{tabular}{|lll|}
\hline \multicolumn{1}{|c}{ Variable } & Odds ratio (95\% CI) & p-value \\
\hline Barthel index & & \\
\hline At admission & $0.997(0.971-1.024)$ & 0.838 \\
\hline At discharge & $1.066(1.026-1.108)$ & 0.001 \\
\hline Pain in rest at admission & $0.795(0.517-1.222)$ & 0.296 \\
\hline Pain during movement & & \\
\hline At admission & $0.943(0.632-1.407)$ & 0.772 \\
\hline At discharge & $2.221(0.812-6.080)$ & 0.120 \\
\hline Treatment strategy & $2.263(0.490-10.441)$ & 0.295 \\
\hline Spinal instability neoplastic score & $0.893(0.624-1.276)$ & 0.534 \\
\hline Motor paralysis & $19.796(1.542-254.072)$ & 0.022 \\
\hline
\end{tabular}

CI, confidence interval.

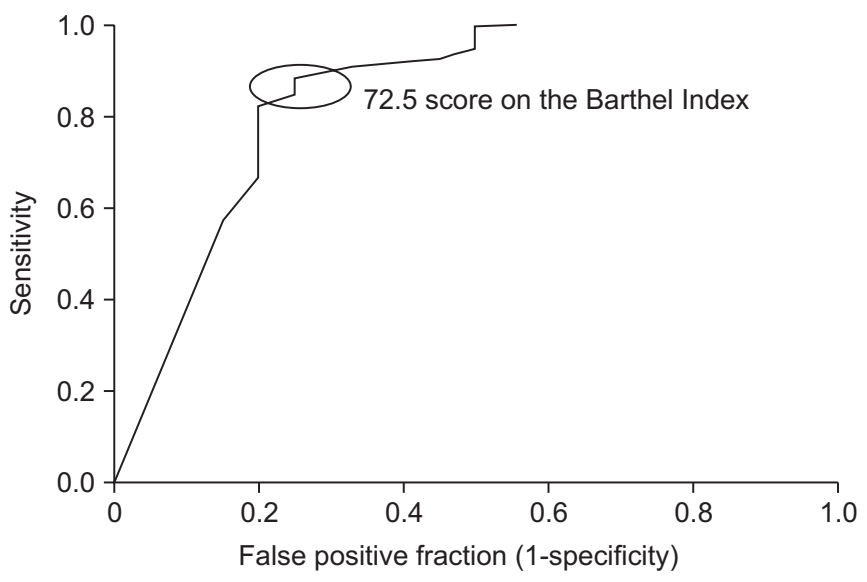

Fig. 1. Receiver operating characteristic curve for prediction of home group. A 72.5 score on the Barthel Index gave a clear cut-off value for the discharges to home.

discharge ADL and motor paralysis were effective predicators concerning the discharge destination of patients with spinal SREs. Even if the patients with SREs showed an ADL decrease on admission, it was still possible that they may have been discharged home following an improvement in their ADL during hospitalization. In addition, motor paralysis affected the discharge destination because motor paralysis results in decreased ADL in patients. Therefore, these results appear to confirm the importance of intensive inpatient rehabilitation for patients with spinal SREs.

Granger et al. [18] reported that a BI score $>40$ in patients with stroke was a good predictor of discharge home; however, the results in this study cannot be readily compared with that study as the diseases differed. The

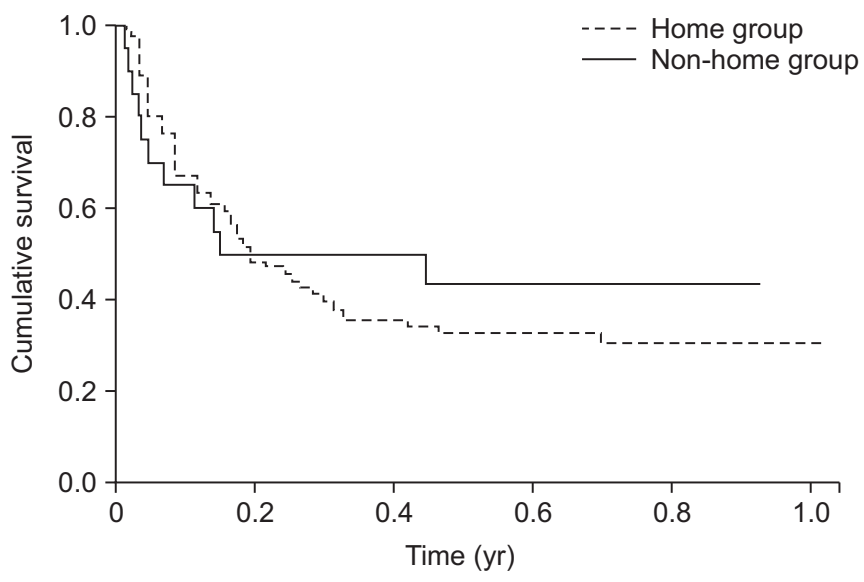

Fig. 2. Kaplan-Meier survival curves for patients with spinal bone metastases by discharge destination.

present study showed that a BI score of 72.5 was a clear cut-off value for home discharge, with a sensitivity of $88.3 \%$, a false-positive rate (1-specificity) of $25.0 \%$, a predictive accuracy of $86.4 \%$, and a positive predictive value of 95.5\%. For patients with SREs to be discharged home, the ADL must reach a certain level, and a BI cut-off value of 72.5 was found to be a predictor of home discharge.

In relation to the length of hospital stay and discharge destination, a study by McKinley et al. [19], involving patients with cancer-related spinal cord compression, found the average length of stay in a rehabilitation unit to be 27 days, with $84 \%$ of patients discharged home. The present study showed similar results, with approximately $86 \%$ of patients with spinal SREs being discharged home, with an average length of hospital stay of 26 days.

With respect to the home environment, the presence 
of family members is likely to be an important element for successful home discharge, since the involvement of family can render discharge more feasible, facilitate patients' functional improvement, and limit discomfort at home [20]. In previous studies, the number of co-resident household members $[15,21,22]$, in addition to ADL (assessed through the BI or the Functional Independence Measure), has been found to be a powerful predictor of home discharge for elderly patients with stroke. The present study showed no significant difference in terms of the number of co-resident household members between the home and non-home groups. In patients with spinal bone metastases, it appears that the discharge destination may depend more on ADL than on the number of co-resident household members.

The present study demonstrated that the non-home group had lower ADL than the home group, but no significant difference in OS was found between the home and non-home groups. Nevertheless, QOL might differ between the two groups. It has been reported that in patients with cancer and with bone metastases, a resistance exercise program improved physical function, physical activity levels, and lean mass [23,24]. These results indicate that patients who wish to return home need to improve their ADL through participating in rehabilitation during hospitalization.

In conclusion, this study showed that discharge ADL and motor paralysis strongly affected the discharge destination of patients with bone metastases, and that a specific ADL level needed to be reached for discharge home. The results of this study are likely to be helpful in determining the discharge destination of patients with bone metastases.

This study had some limitations. Although this study considered the number of co-resident household members as a factor, household income and the relationships of the household members were not examined. Furthermore, the discharge destination of patients may be affected due to psychological conditions such as depression and QOL, comorbidity, and acute length of hospital stay related to the management of differing types of cancer, but these factors were not investigated. In addition, the effect of the total BI score on discharge destination was analyzed without classifying ADL into specific activities such as walking up and down the stairs. Therefore, the kinds of ADL that may have affected the discharge desti- nation could not be determined, and further research is needed to examine these factors.

\section{CONFLICT OF INTEREST}

No potential conflict of interest relevant to this article was reported.

\section{ACKNOWLEDGMENTS}

The authors would like to thank all the patients who participated in this research.

\section{AUTHOR CONTRIBUTION}

Conceptualization: Akezaki Y, Nakata E. Methodology: Akezaki Y, Nakata E. Formal analysis: Akezaki Y. Project administration: Akezaki Y, Nakata E. Data collection: Akezaki Y, Nakata E, Kikuuchi M. Writing - original draft: Akezaki Y, Nakata E, Kikuuchi M, Sugihara S. Writing review and editing: Akezaki Y, Nakata E, Kikuuchi M, Sugihara S. Approval of final manuscript: all authors.

\section{REFERENCES}

1. Coleman RE. Metastatic bone disease: clinical features, pathophysiology and treatment strategies. Cancer Treat Rev 2001;27:165-76.

2. Harrington KD. Orthopedic surgical management of skeletal complications of malignancy. Cancer 1997;80:1614-27.

3. Barron KD, Hirano A, Araki S, Terry RD. Experiences with metastatic neoplasms involving the spinal cord. Neurology 1959;9:91-106.

4. Walsh GL, Gokaslan ZL, McCutcheon IE, Mineo MT, Yasko AW, Swisher SG, et al. Anterior approaches to the thoracic spine in patients with cancer: indications and results. Ann Thorac Surg 1997;64:1611-8.

5. Black P. Spinal metastasis: current status and recommended guidelines for management. Neurosurgery 1979;5:726-46.

6. Schaberg J, Gainor BJ. A profile of metastatic carcinoma of the spine. Spine (Phila Pa 1976) 1985;10:19-20.

7. Stark RJ, Henson RA, Evans SJ. Spinal metastases: a retrospective survey from a general hospital. Brain 1982;105:189-213. 
8. Vieth RG, Odom GL. Extradural spinal metastases and their neurosurgical treatment. J Neurosurg 1965;23:501-8.

9. Bunting RW, Boublik M, Blevins FT, Dame CC, Ford LA, Lavine LS. Functional outcome of pathologic fracture secondary to malignant disease in a rehabilitation hospital. Cancer 1992;69:98-102.

10. Tang V, Harvey D, Park Dorsay J, Jiang S, Rathbone MP. Prognostic indicators in metastatic spinal cord compression: using functional independence measure and Tokuhashi scale to optimize rehabilitation planning. Spinal Cord 2007;45:671-7.

11. Denti L, Agosti M, Franceschini M. Outcome predictors of rehabilitation for first stroke in the elderly. Eur J Phys Rehabil Med 2008;44:3-11.

12. Okuno Y, Miyasaka T, Dobashi K. Factors influencing the outcome of acute rehabilitation: functional independence measure assessment at discharge. J Phys Ther Sci 2012;24:491-4.

13. Fisher CG, DiPaola CP, Ryken TC, Bilsky MH, Shaffrey $\mathrm{CI}$, Berven SH, et al. A novel classification system for spinal instability in neoplastic disease: an evidencebased approach and expert consensus from the Spine Oncology Study Group. Spine (Phila Pa 1976) 2010;35:E1221-9.

14. Mahoney FI, Barthel DW. Functional evaluation: the barthel index. Md State Med J 1965;14:61-5.

15. Borg G. Psychophysical scaling with applications in physical work and the perception of exertion. Scand J Work Environ Health 1990;16 Suppl 1:55-8.

16. Oczkowski WJ, Barreca S. The functional independence measure: its use to identify rehabilitation needs in stroke survivors. Arch Phys Med Rehabil 1993;74:1291-4.
17. Massucci M, Perdon L, Agosti M, Celani MG, Righetti E, Recupero E, et al. Prognostic factors of activity limitation and discharge destination after stroke rehabilitation. Am J Phys Med Rehabil 2006;85:963-70.

18. Granger CV, Dewis LS, Peters NC, Sherwood CC, Barrett JE. Stroke rehabilitation: analysis of repeated Barthel index measures. Arch Phys Med Rehabil 1979;60:14-7.

19. McKinley WO, Conti-Wyneken AR, Vokac CW, Cifu DX. Rehabilitative functional outcome of patients with neoplastic spinal cord compressions. Arch Phys Med Rehabil 1996;77:892-5.

20. Agarwal V, McRae MP, Bhardwaj A, Teasell RW. A model to aid in the prediction of discharge location for stroke rehabilitation patients. Arch Phys Med Rehabil 2003;84:1703-9.

21. Koyama T, Sako Y, Konta M, Domen K. Poststroke discharge destination: functional independence and sociodemographic factors in urban Japan. J Stroke Cerebrovasc Dis 2011;20:202-7.

22. Sommerfeld DK, von Arbin MH. Disability test 10 days after acute stroke to predict early discharge home in patients 65 years and older. Clin Rehabil 2001;15:52834.

23. Cormie P, Newton RU, Spry N, Joseph D, Taaffe DR, Galvao DA. Safety and efficacy of resistance exercise in prostate cancer patients with bone metastases. Prostate Cancer Prostatic Dis 2013;16:328-35.

24. Cormie P, Galvao DA, Spry N, Joseph D, Taaffe DR, Newton RU. Functional benefits are sustained after a program of supervised resistance exercise in cancer patients with bone metastases: longitudinal results of a pilot study. Support Care Cancer 2014;22:1537-48. 